\title{
Effects of collisions on the generation and suppression of temperature anisotropies and the Weibel instability
}

\author{
K. M. Schoeffler $\odot$ and L. O. Silva $\odot$ \\ GoLP/Instituto de Plasmas e Fusão Nuclear, Instituto Superior Técnico, Universidade de Lisboa, 1049-001 Lisboa, Portugal
}

(Received 29 April 2020; accepted 21 July 2020; published 11 August 2020)

\begin{abstract}
The expansion of plasma with nonparallel temperature and density gradients and the generation of a magnetic field via the Biermann battery is modeled using particle-in-cell simulations that include collisional effects via Monte Carlo methods. A scaling of the degree of collisionality shows that an anisotropy can be produced and drive the Weibel instability for gradient scales shorter than the mean free path. For larger collision rates the Biermann battery dominates as the cause of magnetic-field generation. When the most energetic particles remain collisionless the Nernst effect causes the Biermann field to be dragged with the heat flux piled up and enhanced.
\end{abstract}

DOI: 10.1103/PhysRevResearch.2.033233

\section{INTRODUCTION}

Identifying the mechanisms responsible for the generation of various magnetic fields present throughout the universe is a major topic of study in astrophysics [1]. Two common candidates are the Biermann battery [2,3], which is driven by violent interactions with unmagnetized plasmas that leave the temperature and density gradients misaligned, and the Weibel instability [4], driven by temperature anisotropies. The Weibel instability, in particular, is only possible in collisionless systems, often found in astrophysics due to high temperatures and low densities of plasmas in space. In laser-plasma interaction experiments on earth, both the Biermann battery [3,5-7] and the Weibel instability [7-10] play an important role. The sudden heating of the plasma by a laser leads to the gradients required for the Biermann battery, and although the densities can be large, the temperatures can be sufficiently high that the plasma is collisionless, can become anisotropic and, thus, become unstable to the Weibel instability.

A natural question is as follows: What level of collisionality is required for the Weibel instability to be suppressed? It was shown in Refs. [11,12] that, for a collisionless system, the Weibel instability is the dominant magnetic field for sufficiently large gradient scales $L / d_{e}>100$, where $L$ is the temperature or density gradient length scale, $d_{e}=c / \omega_{p e}$ is the electron skin depth, $\omega_{p e}=\sqrt{4 \pi n_{e} e^{2} / m_{e}}$ is the plasma frequency, $m_{e}, e$, and $n_{e}$ are the respective electron mass, charge, and density, and $c$ is the speed of light. However, this is no longer the case for a sufficiently collisional plasma (e.g., Ref. [13]), where the Biermann field becomes the dominant field. Here, we show, using particle-in-cell (PIC) simulations,

Published by the American Physical Society under the terms of the Creative Commons Attribution 4.0 International license. Further distribution of this work must maintain attribution to the author(s) and the published article's title, journal citation and DOI. what level of collisionality is required for the Biermann field to dominate over the Weibel instability.

Although the original Weibel formulation assumes a collisionless plasma, the instability has been formulated for a semicollisional system showing a dependence on the electron collision rate $v_{e}[14,15]$,

$$
\gamma_{W}=\gamma_{W 0}-\frac{A}{1+A} v_{e}
$$

Here, $\gamma_{W 0}$ is the collisionless growth rate of the Weibel instability [4], which depends on the perpendicular electron temperature $T_{e \perp}, \omega_{p e}$, and the temperature anisotropy $A=$ $T_{e \|} / T_{e \perp}-1$, where $T_{e \|}$ and $T_{e \perp}$ are the two temperatures in a bi-Maxwellian distribution. Here, parallel is defined by the direction that has a different temperature, and we have assumed $T_{e \|}>T_{e \perp}$. The collisionless growth rate can be calculated using the dispersion relation,

$$
k^{2} c^{2}+\gamma_{W 0}^{2}-\omega_{p e}^{2}[A+(1+A) \xi Z(\xi)]=0,
$$

where one can solve for the fastest growing wave-number $k=$ $k_{\max }$. Here, $\xi \equiv i \gamma_{W 0} / k \sqrt{T_{e \perp} / 2 m_{e}}$ and $Z(\xi)$ is the plasma dispersion function. The collision rate is $v_{e}=v_{0}(1+Z)$ where

$$
v_{0}=\sqrt{\frac{1}{m_{e}}} \frac{4 \pi n_{e} e^{4}}{T_{e}^{3 / 2}} \ln \Lambda_{C},
$$

$T_{e} \equiv\left(2 T_{e \perp}+T_{e \|}\right) / 3$ is the electron temperature, $\ln \Lambda_{C}$ is the Coulomb logarithm, and $Z$ is the degree of ionization. $v_{e}$ can be divided into electron-electron collisions $v_{e e}=v_{0}$, and electron-ion collisions $v_{e i}=v_{0} Z$.

In this paper, we will focus on an initially isotropic Maxwellian plasma where the temperature anisotropy is generated self-consistently via temperature gradients. Although the plasma does not remain strictly bi-Maxwellian, this approximation continues to produce growth rates consistent with theory. In principle, the arguments made here may hold, at least, qualitatively, in more non-Maxwellian systems, e.g., 
distributions with a hot streaming population of electrons [16-18].

It turns out that, in the regime of this paper, among many others, the modifications to $\gamma_{W}$ due to collisions are not relevant. The growth rate drops to zero even when these modifications are negligible because the instability is driven by $A$ and collisions cause $A$ to decay. The ratio of the collisional term in Eq. (1) to $\gamma_{W 0}$ is $\sim v_{e} /\left(\omega_{p} v_{T} / c\right)=\left(d_{e} / L_{T}\right) v_{e} L_{T} / v_{T}$, where $L_{T} \equiv T_{e} / d T_{e} / d x$ is the temperature gradient scale and $v_{T}=\sqrt{T_{e} / m_{e}}$ is the thermal velocity. As long as $L_{T} \gg d_{e}$, the collision term can be neglected unless $v_{e} L_{T} / v_{T} \gg 1$, which we will show corresponds to a regime where the Weibel instability is not expected to develop as collisions prevent $A$ from building up.

The growth rate drops to zero when the timescale of the anisotropy generation $t_{A g}$ reaches the timescale of collisional relaxation time of the anisotropy $t_{A r}$. Reference [19] showed that a gradient in an isotropic Maxwellian temperature leads to a temperature anisotropy $A$, saturating at a timescale $t_{A g} \approx$ $L_{T} / v_{T}$ with a value of $A \sim 1$. References [20,21] showed that the relaxation rate is

$$
v_{A} \equiv \frac{1}{A} \frac{d A}{d t}=-v_{T}(A+3),
$$

where

$$
\nu_{T}=\frac{2 v_{0}\left(T_{e \perp}\right)}{15 \sqrt{\pi}}(1+\sqrt{2} Z) F_{2,3 / 2,7 / 2}[A /(A+1)],
$$

$v_{0}\left(T_{e \perp}\right)$ is $v_{0}$ replacing $T_{e}$ with $T_{e \perp}, F_{2,3 / 2,7 / 2}(x)=15 / 4[-3+$ $(x+3) \phi(x)] x^{-2}$ is the Gaussian hypergeometric function, and

$$
\phi(x)= \begin{cases}\frac{\tan ^{-1}(\sqrt{x})}{\sqrt{x}}, & \text { if } x>0, \\ 1, & \text { if } x=0, \\ \frac{\tanh ^{-1}(\sqrt{-x})}{\sqrt{-x}}, & \text { if } x<0 .\end{cases}
$$

The factors of 1 and $\sqrt{2} Z$ are a result of the respective electron-electron and electron-ion collisions. One finds
$v_{A} \sim v_{e}$ using Eqs. (4) and (5) since $F_{2(3 / 2)(7 / 2)}(x)$ is a constant of order unity (approaching 1 for small $A$ ) as long as $A \lesssim 1$. Remember the anisotropy is expected to reach a maximum of $A \sim 1$. The Weibel instability is, thus, suppressed if

$$
\frac{t_{A g}}{t_{A r}}=\frac{v_{A} L_{T}}{v_{T}} \sim \frac{v_{e} L_{T}}{v_{T}}>1,
$$

i.e., if the gradient scale is bigger than the mean free path of an electron.

Even if the Weibel instability is fully suppressed, it has been suggested that an instability known as the thermomagnetic instability [22] may also generate filamentary magnetic fields due to a parallel density and temperature gradient due to a combination of the Righi-Leduc and Biermann battery effects for collisional systems. However, Ref. [23] showed that this instability is suppressed due to the Nernst effect and that the growth of the Biermann battery is reduced. The Nernst effect, when only the most energetic electrons are frozen into the magnetic field, is expected to drag Biermann generated fields in the direction of heat flux allowing them to pile up [24-30].

\section{SIMULATION SETUP}

In order to verify and quantify the scalings of Eq. (7), we performed several simulations of an expanding bubble of plasma using the OSIRIS framework [31,32], whereas including collisional effects [33,34], and varying the collisionality $v_{0} L_{T} / v_{T}$ (via the density). The bubble has a peak density $n_{0}$ and expands into a background $n_{b}=0.1 n_{0}$ with a peak initial temperature at the center of the box $T_{e 0} / m_{e} c^{2}=0.04$ (20.44 $\mathrm{keV}$ ) varying only along the $x$ direction to a background temperature $T_{e b}=0.0025 T_{e 0}(51.1 \mathrm{eV})$, and a realistic mass ratio $m_{i} / m_{e}=1836$, the same as the simulations in Ref. [12] with

$$
\begin{aligned}
n & = \begin{cases}\left(n_{0}-n_{b}\right) \cos \left(\pi r / 2 L_{T}\right)^{2}+n_{b}, & \text { if } r<L_{T}, \\
n_{b}, & \text { otherwise, }\end{cases} \\
v_{T e} & =\left\{\begin{array}{ll}
\left(v_{T e 0}-v_{T e b}\right) \cos \left(\pi|x| / 2 L_{T}\right)^{2}+v_{T e b}, & \text { if }|x|<L_{T}, \\
v_{T e b}, & \text { otherwise, }
\end{array} \text { where } r=\sqrt{x^{2}+\left(L_{T} / L_{n} y\right)^{2},}\right.
\end{aligned}
$$

and $L_{n}=L_{T} / 2 . n_{0}$ is the reference density used to define $\omega_{p e}$ and $d_{e}$ and is used along with the reference temperature $T_{e 0}$ to calculate $v_{T}$ and $v_{0}$. The distributions of density and temperature are shown in Fig. 1 with the gradients highlighted. Unless otherwise specified, each simulation uses 198 particles per cell on a $12000 \times 12000$ grid $\left(1500 \times 1500 d_{e}^{2}\right)$. The simulations are run for $1800 \omega_{p e}^{-1}$, with a time-step $d t=0.07 \omega_{p e}^{-1}$. The Coulomb logarithm $\ln \Lambda_{C}$ is calculated automatically depending on the local parameters. Only the collisions between electrons and ions are included. There is an eight-point average for diagnostic purposes over the generated magnetic fields.
In realistic experimental setups where collisions become important, the temperatures are lower, and the system sizes are larger than we simulate here. As these parameters are more computationally expensive, we, instead, vary the density, ranging between $1 \times 10^{18}$ and $1 \times 10^{28} \mathrm{~cm}^{-3}$ separated by factors of 100 for the simulations with $L_{T} / d_{e}=400$, allowing for a scaling to realistic parameters. For example, our case with $v_{0} L_{T} / v_{T}=0.837$ corresponds to a density of $n_{0}=1 \times 10^{24} \mathrm{~cm}^{-3}$, and, therefore, $L_{T}=2.13 \mu \mathrm{m}$ (for $L_{T} / d_{e}=400$ ). However, the same result should occur for an equal $v_{0} L_{T} / v_{T}$ with lower density but larger $L_{T}$ and lower temperature. Note that for the parameters that we simulate, 


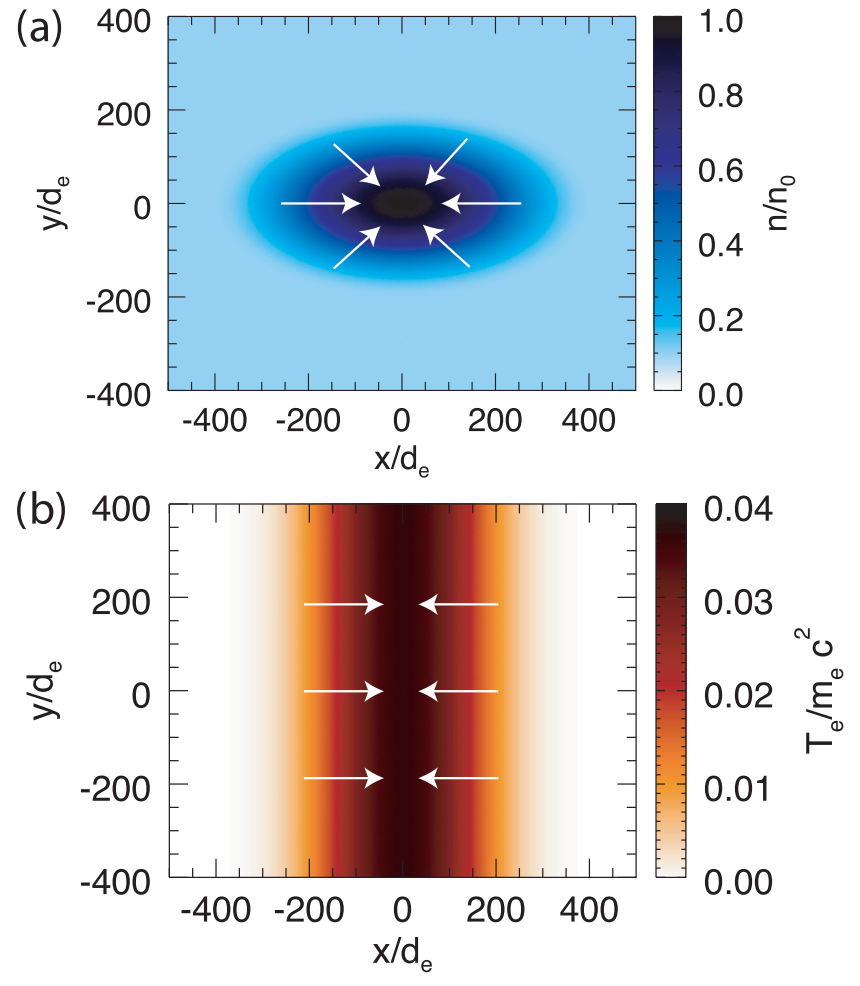

FIG. 1. Map of the initial (a) density $n$ and (b) electron temperature $T_{e}$. The gradients are highlighted in white.

$\ln \Lambda_{C}$ varies significantly due to the small values if $\Lambda_{C}$. Once $v_{T} / c$ exceeds $2 \alpha\left(T_{e}>108 \mathrm{eV}\right)$, where $\alpha$ is the fine-structure constant, $\Lambda_{C}$ grows more slowly with respect to temperature. Therefore, for a given collision rate, $\Lambda_{C}$ is smaller for higher temperatures. Furthermore, for larger system sizes, equal collisionalities occur at smaller collision rates and, thus, at larger $\Lambda_{C}$.

In our simulations, the velocity distribution does not necessarily remain bi-Maxwellian, and we measure the anisotropy using the temperature tensor $T_{i j} \equiv \int\left(u_{i} u_{j} / \gamma\right) f(u) / \int f(u)$ calculated in the species rest frame. $u_{i}$ is the proper velocity, $\gamma=\sqrt{1+u^{2}}$, and $f(u)$ is the velocity distribution function. $T_{e \|}$ and $T_{e \perp}<T_{e \|}$ are eigenvalues of the temperature tensor. We only consider the in-plane temperatures and assume the out-of-plane temperature is also $T_{e \perp}$, which has been verified for our simulations to be a reasonable assumption.

\section{SIMULATION RESULTS}

In Fig. 2, the out-of-plane magnetic-field $B_{z}$ for three representative collisionalities is presented. The first case [Fig. 2(a)] is like the previous collisionless studies where the Weibel magnetic field dominates compared to the fields due to the Biermann battery. Only the growth rate and the strength of the saturated field are modified by the collisions. The second case [Fig. 2(b)] is the transition scale where the Weibel fields are suppressed but still visible, and the Biermann field is the dominant field. In the third case [Fig. 2(c)], only the most energetic electrons remain collisionless, leading to the pileup of Biermann generated fields via the Nernst effect [24-30]. We thus demonstrate the Nernst effect using PIC
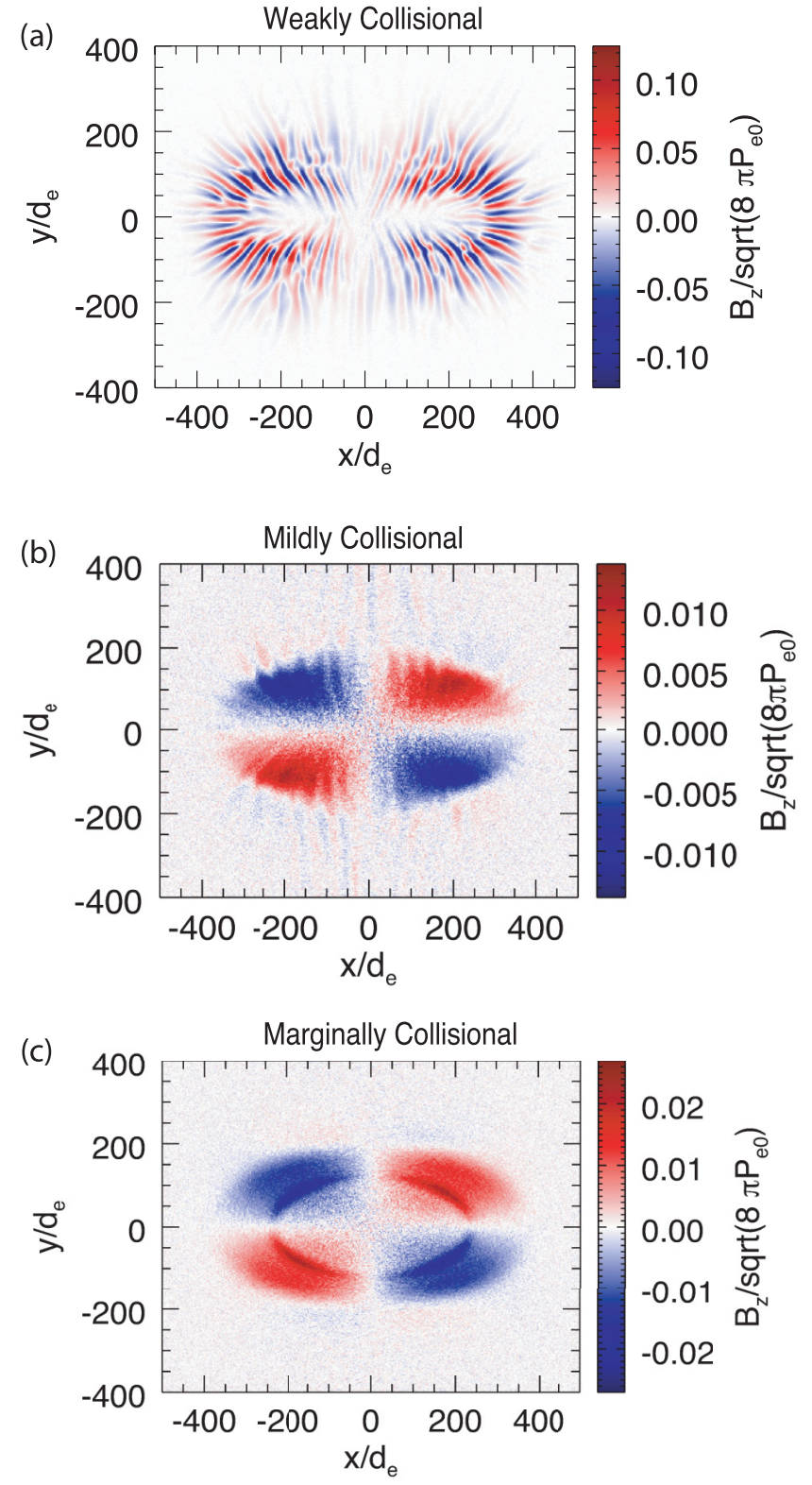

FIG. 2. Map of magnetic-field $B_{z}$ at $t \omega_{p e}=1797.6$. The top panel shows the simulation with a collisionality $v_{0} L_{T} / v_{T}=0.837$ where the Weibel instability still exists but grows slower and saturates at a lower intensity field. The middle panel shows a simulation with $v_{0} L_{T} / v_{T}=5.32$ where the Weibel instability is significantly damped, and the Biermann field is visible. The bottom panel shows a simulation with $v_{0} L_{T} / v_{T}=26.5$ where there are no traces of the Weibel instability, and a pileup of magnetic flux dragged by the Nernst effect is present.

simulations. A full demonstration beyond these observations of the magnetic pileup remains to be presented.

We can make a prediction where to expect the electron Weibel instability, depending on the gradient length scale, temperature, density, and charge state of the ions. The transition occurs when $v_{e} L_{T} / v_{T} \approx 1$ as predicted from Eq. (7). The three cases in Fig. 2 occur at $v_{0} L_{T} / v_{T}=0.837$, 5.32 , and 26.5 , respectively. We calculate the local $v_{A}$ at the location where the instability occurs in the collisionless 


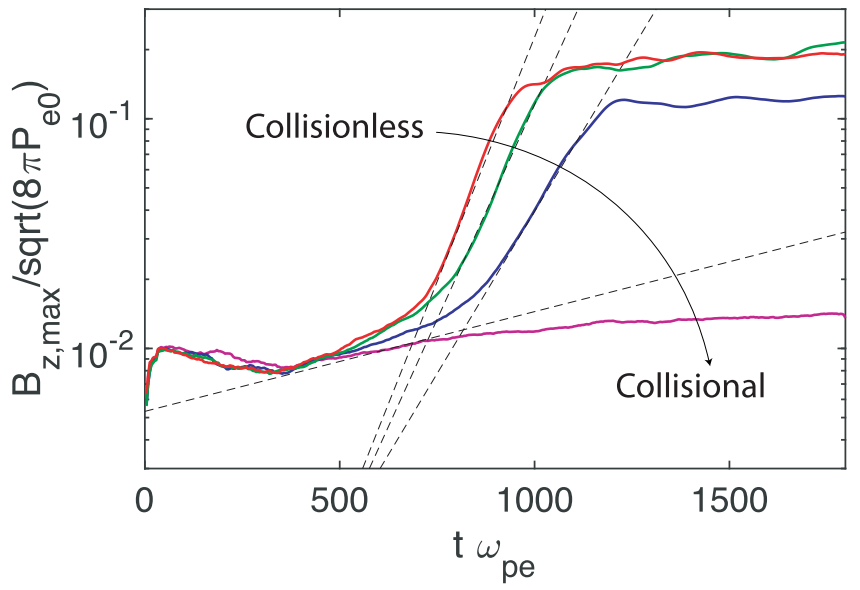

FIG. 3. Evolution of the maximum magnetic-field $B_{z, \max }$ (produced via the Weibel instability) vs time for simulations with various collisionalities $v_{0} L_{T} / v_{T}=0.00175$ (red), 0.114 (green), 0.837 (blue), and 5.32 (magneta). The measured slopes (at $t_{c}$ where the slope reaches its maximum) occurring at $t_{c} \omega_{p e}=907,946.5,1047$, and 600 are shown in dashed black.

case $\left(x / d_{e}, y / d_{e}=150,100\right)$ by measuring the parameters averaged within a box of $20 \times 10 d_{e}^{2}$ at time $t_{c} \omega_{p e}=907$ when the measured growth rate reaches its maximum. Here, $T_{e \perp, \mathrm{loc}} / m_{e} c^{2}=0.0244, A=0.56, n_{\mathrm{loc}} / n_{0}=0.385$, and the gradient length scale $L_{T, \text { loc }} / L_{T}=0.2563$. We, thus, find the respective cases occur at $v_{A, \text { loc }} L_{T, \text { loc }} / v_{T, \text { loc }}=2.09,13.5$, and 63.7. We, therefore, confirm that the Weibel instability is suppressed when the anisotropy relaxation time is smaller than the generation time $\left(t_{A r}>t_{A g}\right)$, but it is not completely suppressed until $t_{A r} \gg t_{A g}$.

The transition to a regime where no Weibel exists occurs in the simulation with $v_{0} L_{T} / v_{T}=5.32$ [Fig. 2(b)]. This simulation has no electron-electron collisions, $Z=1$, and the local $L_{T, \text { loc }}=55 \mathrm{~nm}, \ln \Lambda_{C}=4.0$, and $v_{T, \text { loc }} / c=0.170\left(T_{e, \text { loc }}=\right.$ $14.8 \mathrm{keV})$. Using this numerical value of $v_{0} L_{T} / v_{T}$ and the scaling from Eq. (7), our simulation result can be scaled to more experimentally relevant densities and temperatures, and the general transition density can be expressed in an engineering formula,

$$
\begin{aligned}
n_{t r, \text { loc }}= & 5.42 \times 10^{21} \mathrm{~cm}^{-3}\left(\frac{T_{e, \text { loc }}}{1.0 \mathrm{keV}}\right)^{2}(1+\sqrt{2} Z)^{-1} \\
& \times\left(\frac{L_{T, \text { loc }}}{1.0 \mu m}\right)^{-1}\left(\frac{\ln \Lambda_{C}}{10.0}\right)^{-1} .
\end{aligned}
$$

The other two cases correspond to a density $n_{\mathrm{loc}}=0.1 n_{t r \text {, loc }}$ [Fig. 2(a)] and $10 n_{t r \text {,loc }}$ [Fig. 2(c)].

We show the effects of collisions on the Weibel growth in Fig. 3, plotting the evolution of the maximum magnetic field and exponential fits of the growth rate. There is a significant change in growth rate between the essentially collisionless case at $v_{0} L_{T} / v_{T}=0.00175$ and $v_{0} L_{T} / v_{T}=$ $0.837\left(0.0098-0.0065 \omega_{p e}\right)$. The growth rate effectively goes to zero in the case with $v_{0} L_{T} / v_{T}=5.32$. The measured growth rates of the magnetic field for each simulation are reported in Table I along with the local parameters used to calculate the theoretical Weibel growth rate. Table II shows
TABLE I. Measured growth rate $\gamma_{m}$ and parameters determining the theoretical growth rate $\gamma_{t}$ at $k=k_{\max }$ [see Eq. (1)] at the location where the instability occurs $\left(x / d_{e}, y / d_{e}=150,100\right)$ averaged within a box of $20 \times 10 d_{e}^{2}$ at time $t_{c}$ where the measured growth rate reaches its maximum. The local density is $0.385 n_{0}$. For $v_{0} L_{T} / v_{T}=5.32$, the measured growth rate can be considered 0 .

\begin{tabular}{lcrcccc}
\hline \hline$v_{0} L_{T} / v_{T}$ & $v_{0} / \omega_{p e}$ & $t_{c} \omega_{p e}$ & $\gamma_{m} / \omega_{p e}$ & $A$ & $T_{e \perp} / m_{e} c^{2}$ & $\gamma_{t} / \omega_{p e}$ \\
\hline 0.00175 & $8.8 \times 10^{-7}$ & 907.0 & 0.0098 & 0.56 & 0.0244 & 0.0095 \\
0.0145 & $7.2 \times 10^{-6}$ & 900.6 & 0.0111 & 0.56 & 0.0245 & 0.0096 \\
0.114 & $5.7 \times 10^{-5}$ & 946.5 & 0.0086 & 0.57 & 0.0241 & 0.0097 \\
0.837 & $4.2 \times 10^{-4}$ & 1047.0 & 0.0065 & 0.47 & 0.0225 & 0.0072 \\
5.32 & $2.7 \times 10^{-3}$ & 600.0 & 0.0001 & 0.02 & 0.0224 & 0.0000 \\
26.5 & $1.3 \times 10^{-2}$ & 500.0 & 0.0001 & 0.02 & 0.0217 & -0.000 \\
\hline \hline
\end{tabular}

the measured wave number of the instability, the predicted fastest growing wave numbers, and the growth rates calculated using these wave numbers, providing evidence that the observed filaments are due to Weibel instability.

The growth rate of the Weibel instability depends on $A$, which depends on the collisionality as collisions inhibit the anisotropy growth. Due to the exponential decay of $A$ predicted by Eq. (4) for a constant $v_{A}$, a good approximation of the $A$ dependence on the local collisionality is as follows:

$$
A \approx A_{0} \exp \left(-\frac{v_{A, \mathrm{loc}} L_{T, \mathrm{loc}}}{4 v_{T, \mathrm{loc}}}\right)
$$

In addition to the simulations presented so far with $L_{T} / d_{e}=400$, we have simulated several more simulations with $L_{T} / d_{e}=200$ (half the system size with constant resolution), where we have also measured the growth rate. In Fig. 4, the measured growth rates normalized to the collisionless growth rates are presented as a function of the local collisionality $v_{A, \text { loc }} L_{T, \text { loc }} / v_{T, \text { loc }} \cdot v_{A \text {, loc }}$ is calculated as previously assuming a constant anisotropy $A_{0}=0.56$ and perpendicular temperature $T_{e \perp, \mathrm{loc}} / m_{e} c^{2}=0.0244$. A theoretical prediction for the growth rate is given by the Weibel growth rate using the anisotropy from Eq. Eq. (10) (black curve in Fig. 4), which agrees with the measured results. Figure 4 also gives evidence that this scaling with collisionality is independent of $L_{T}$ for constant $v_{0} L_{T} / v_{T}$.

TABLE II. The measured wave-numbers $k$, the theoretical fastest growing mode $k_{\max }$, and the theoretical growth rates $\gamma_{t}$ using Eq. (1) given these wave numbers.

\begin{tabular}{lcccc}
\hline \hline$\nu_{0} L_{T} / v_{T}$ & $k d_{e}$ & $k_{\max } d_{e}$ & $\gamma_{t}(k) / \omega_{p e}$ & $\gamma_{t} / \omega_{p e}$ \\
\hline 0.00175 & 0.281 & 0.2523 & 0.0094 & 0.0095 \\
0.0145 & 0.204 & 0.2523 & 0.0091 & 0.0096 \\
0.114 & 0.200 & 0.2546 & 0.0091 & 0.0097 \\
0.837 & 0.208 & 0.2312 & 0.0071 & 0.0072 \\
5.32 & 0.080 & 0.0502 & -0.000 & 0.0000 \\
26.5 & $\mathrm{~N} / \mathrm{A}$ & 0.0502 & $\mathrm{~N} / \mathrm{A}$ & -0.000 \\
\hline \hline
\end{tabular}




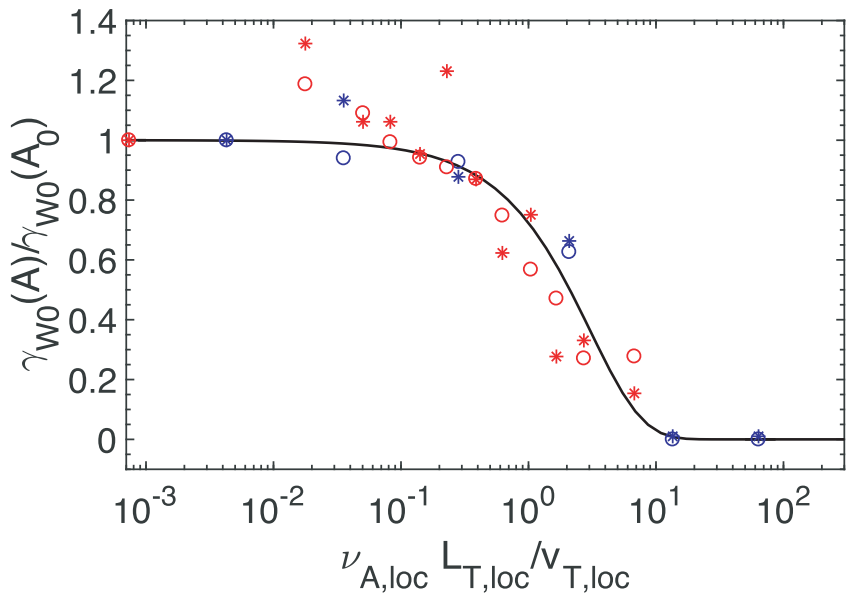

FIG. 4. Measured growth rate of the Weibel instability normalized to the growth rate measured in the collisionless case for a range of simulations with different collisionalities. Simulations with $L_{T} / d_{e}=400$ (200) are indicated in blue (red). Circles are calculated from magnetic-field energy, and stars are calculated from the maximum magnetic field. A theoretical estimate of the growth rate is plotted in black using the anisotropy from Eq. (10).

\section{CONCLUSION}

Using particle-in-cell simulations, we have placed a limit where collisions will inhibit the generation of the electron Weibel instability in the expansion of a hot plasma when $v_{e} L_{T} / v_{T} \sim 1$. Whereas in Ref. [11] it was shown that magnetic fields from the Weibel instability will be larger than the Biermann field for $L_{T} / d_{e}>100$, we now show this additional limit due to collisions where the Biermann field again dominates.

Although the simulations presented here are all two dimensional (2D), the results should not differ greatly in three dimensions (3D). For the collisionless case, a 3D simulation showed similar results for $L_{T} / d_{e}=50$ [12]. For larger system sizes, we expect Weibel filaments with wave numbers also out of the 2D simulation plane, but besides that, the results should remain similar to $2 \mathrm{D}$.

We do not observe the thermomagnetic instability, confirming Ref. [23], but we also do not observe any of the predicted reduction of the Biermann battery growth. This is likely because we start from a Maxwellian distribution where the Biermann battery should grow rather than evolve to a such a state by plasma heating and expansion.

This still remains a simplified model and assumes that the laser interaction will generate these temperature gradients on a quick enough timescale that this model is valid. The effects of the laser magnetic fields and heating processes often occur at the same time as the Biermann and Weibel magnetic fields grow. It has been shown that for an intense short pulse laser where the plasma becomes relativistically hot, the Weibel field can be observed [35].

\section{ACKNOWLEDGMENT}

This work was supported by the European Research Council (ERC-2015-AdG Grant No. 695088).
[1] R. M. Kulsrud and E. G. Zweibel, Rep. Prog. Phys. 71, 046901 (2008).

[2] L. Biermann, Z. Naturforsch. 5a, 65 (1950).

[3] J. A. Stamper, K. Papadopoulos, R. N. Sudan, S. O. Dean, and E. A. McLean, Phys. Rev. Lett. 26, 1012 (1971).

[4] E. S. Weibel, Phys. Rev. 114, 18 (1959).

[5] P. M. Nilson, L. Willingale, M. C. Kaluza, C. Kamperidis, S. Minardi, M. S. Wei, P. Fernandes, M. Notley, S. Bandyopadhyay, M. Sherlock, R. J. Kingham, M. Tatarakis, Z. Najmudin, W. Rozmus et al., Phys. Rev. Lett. 97, 255001 (2006).

[6] C. K. Li, F. H. Seguin, J. A. Frenje, J. R. Rygg, R. D. Petrasso, R. P. J. Town, O. L. Landen, J. P. Knauer, and V. A. Smalyuk, Phys. Rev. Lett. 99, 055001 (2007).

[7] N. L. Kugland, D. D. Ryutov, P.-Y. Chang, R. P. Drake, G. Fiksel, D. H. Froula, S. H. Glenzer, G. Gregori, M. Grosskopf, M. Koenig, Y. Kuramitsu, C. Kuranz, M. C. Levy, E. Liang et al., Nature (London) 8, 809 (2012).

[8] C. M. Huntington, F. Fiuza, J. S. Ross, A. B. Zylstra, R. P. Drake, D. H. Froula, G. Gregori, N. L. Kugland, C. C. Kuranz, M. C. Levy, C. K. Li, J. Meinecke, T. Morita, R. Petrasso, C. Plechaty, B. A. Remington, D. D. Ryutov, Y. Sakawa, A. Spitkovsky, H. Takabe, and H. S. Park, Nat. Phys. 11, 173 (2015).

[9] S. Göde, C. Rödel, K. Zeil, R. Mishra, M. Gauthier, F.-E. Brack, T. Kluge, M. J. MacDonald, J. Metzkes, L. Obst, M. Rehwald, C. Ruyer, H.-P. Schlenvoigt, W. Schumaker, P. Sommer,
T. E. Cowan, U. Schramm, S. Glenzer, and F. Fiuza, Phys. Rev. Lett. 118, 194801 (2017).

[10] G. K. Ngirmang, J. T. Morrison, K. M. George, J. R. Smith, K. D. Frische, C. Orban, E. A. Chowdhury, and W. M. Roquemore, Sci. Rep. 10, 9872 (2020).

[11] K. M. Schoeffler, N. F. Loureiro, R. A. Fonseca, and L. O. Silva, Phys. Rev. Lett. 112, 175001 (2014).

[12] K. M. Schoeffler, N. F. Loureiro, R. A. Fonseca, and L. O. Silva, Phys. Plasmas 23, 056304 (2016).

[13] W. Fox, J. Matteucci, C. Moissard, D. B. Schaeffer, A. Bhattacharjee, K. Germaschewski, and S. X. Hu, Phys. Plasmas 25, 102106 (2018)

[14] J. M. Wallace, J. U. Brackbill, C. W. Cranfill, D. W. Forslund, and R. J. Mason, Phys. Fluids 30, 1085 (1987).

[15] J. M. Wallace and E. M. Epperlein, Phys. Fluids B 3, 1579 (1991).

[16] L. Romagnani, A. P. L. Robinson, R. J. Clarke, D. Doria, L. Lancia, W. Nazarov, M. M. Notley, A. Pipahl, K. Quinn, B. Ramakrishna, P. A. Wilson, J. Fuchs, O. Willi, and M. Borghesi, Phys. Rev. Lett. 122, 025001 (2019).

[17] S. Krishnamurthy, K. Makur, and B. Ramakrishna, Laser Part. Beams 38, 152 (2020).

[18] C. Ruyer, S. Bolaños, B. Albertazzi, S. N. Chen, P. Antici, J. Böker, V. Dervieux, L. Lancia, M. Nakatsutsumi, L. Romagnani, R. Shepherd, M. Swantusch, M. Borghesi, O. Willi, H. Pépin, M. Starodubtsev, M. Grech, C. Riconda, L. Gremillet, and J. Fuchs, Nat. Phys. (2020). 
[19] K. M. Schoeffler and L. O. Silva, Plasma Phys. Controlled Fusion 60, 014048 (2018).

[20] P. Hellinger and P. M. Travnicek, Phys. Plasmas 16, 054501 (2009).

[21] P. Hellinger and P. M. Travnicek, J. Comput. Phys. 229, 5432 (2010).

[22] D. A. Tidman and R. A. Shanny, Phys. Fluids 17, 1207 (1974).

[23] M. Sherlock and J. J. Bissell, Phys. Rev. Lett. 124, 055001 (2020).

[24] A. Nishiguchi, T. Yabe, M. G. Haines, M. Psimopoulos, and H. Takewaki, Phys. Rev. Lett. 53, 262 (1984).

[25] T. H. Kho and M. G. Haines, Phys. Rev. Lett. 55, 825 (1985).

[26] C. P. Ridgers, R. J. Kingham, and A. G. R. Thomas, Phys. Rev. Lett. 100, 075003 (2008).

[27] L. Willingale, A. G. R. Thomas, P. M. Nilson, M. C. Kaluza, S. Bandyopadhyay, A. E. Dangor, R. G. Evans, P. Fernandes, M. G. Haines, C. Kamperidis, R. J. Kingham, S. Minardi, M. Notley, C. P. Ridgers, W. Rozmus, M. Sherlock, M. Tatarakis, M. S. Wei, Z. Najmudin, and K. Krushelnick, Phys. Rev. Lett. 105, 095001 (2010).

[28] L. Willingale, P. M. Nilson, M. C. Kaluza, A. E. Dangor, R. G. Evans, P. Fernandes, M. G. Haines, C. Kamperidis, R. J. Kingham, C. P. Ridgers, M. Sherlock, A. G. R. Thomas,
M. S. Wei, Z. Najmudin, K. Krushelnick, S. Bandyopadhyay, M. Notley, S. Minardi, M. Tatarakis, and W. Rozmus, Phys. Plasmas 17, 043104 (2010).

[29] A. S. Joglekar, A. G. R. Thomas, W. Fox, and A. Bhattacharjee, Phys. Rev. Lett. 112, 105004 (2014).

[30] A. S. Joglekar, C. P. Ridgers, R. J. Kingham, and A. G. R. Thomas, Phys. Rev. E 93, 043206 (2016).

[31] R. A. Fonseca, L. O. Silva, F. S. Tsung, V. K. Decyk, W. Lu, C. Ren, W. B. Mori, S. Deng, S. Lee, T. Katsouleas, and J. C. Adam, in OSIRIS: A Three-Dimensional, Fully Relativistic Particle in Cell Code for Modeling Plasma Based Accelerators, edited by P. M. A. Sloot, A. G. Hoekstra, C. J. K. Tan, and J. J. Dongarra, Lecture Notes in Computer Science Vol. 2331 (Springer, Berlin/Heidelberg, 2002), p. 342.

[32] R. A. Fonseca, S. F. Martins, L. O. Silva, J. W. Tonge, F. S. Tsung, and W. B. Mori, Plasma Phys. Controlled Fusion 50, 124034 (2008).

[33] F. Fiuza, M. Marti, R. A. Fonseca, L. O. Silva, J. Tonge, J. May, and W. B. Mori, Plasma Phys. Controlled Fusion 53, 074004 (2011).

[34] F. Peano, M. Marti, L. O. Silva, and G. Coppa, Phys. Rev. E 79, 025701(R) (2009).

[35] N. Shukla, K. Schoeffler, E. Boella, J. Vieira, R. Fonseca, and L. O. Silva, Phys. Rev. Res. 2, 023129 (2020). 